\title{
Plasmid pBR322 and nonlinear conformational distortions (kinks)
}

\author{
Yakushevich L.V. ${ }^{1^{*}}$, Krasnobaeva L.A., ${ }^{2, * *}$ \\ ${ }^{1}$ Institute of Cell Biophysics of the Russian Academy of Sciences, Pushchino, \\ Moscow region, Russia \\ ${ }^{2}$ Siberian State Medical University, Tomsk, Russia \\ ${ }^{3}$ Tomsk State University, Tomsk, Russia
}

\begin{abstract}
Plasmid pBR322 containing two coding regions in the matrix chain is a convenient object to study the DNA nonlinear dynamics that is known to play an important role in the processes of transcription, replication, denaturation and transmission of structural changes and information along the DNA molecule. The aim of the present work is to study by the methods of mathematical modeling the dynamics of local conformational distortions - kinks, in the plasmid pBR322. To calculate the dynamic characteristics of the kinks, we applied the method of McLaughlin-Scott, complemented by the block method. This permitted us to model kinks as quasi-particles moving in the potential field of the plasmid. We calculated the time dependences of the kink energy, velocity and coordinate. Calculations were made for three different values of the initial kink velocity: $150 \mathrm{~m} / \mathrm{s}, 1650 \mathrm{~m} / \mathrm{s}$ and $1879 \mathrm{~m} / \mathrm{s}$. The results obtained presented in the form of 3D trajectories and their projections, showed that the necessary condition for kink passing the entire plasmid is the enough large value of the initial kink velocity: $v \geq 1656.66 \mathrm{~m} / \mathrm{c}$ which is, however, less than the sound velocity $(1904.60 \mathrm{~m} / \mathrm{c})$.
\end{abstract}

Key words: plasmid pBR322, kinks, McLaughlin-Scott equation, 3D trajectories.

\section{INTRODUCTION}

The DNA molecule is widely considered as a complex dynamic system that has a variety of different types of internal motions caused by thermal fluctuations, by collisions with the small molecules of the solution or by interactions with proteins. Translational motions of individual atoms, solid-like motions of the atomic groups (sugars, bases and phosphate groups), complex motions of the whole fragments of the DNA polynucleotide chains to form nonlinear conformation distortions of the double helix, are among them. It is believed that there is a relation between the DNA internal dynamics and function which is known to be determined by the nucleotide sequence and the arrangement of the main functionally important regions. To find this relation is one of the most interesting and perspective tasks of DNA science.

In this paper, we pay attention to the movements of small (several base pairs in length) distortions of the DNA structure, within which the hydrogen bonds are broken. These distortions are named the DNA open states [1]. It is assumed that they play an important role in the processes of transcription [2-4], replication [5, 6], denaturation [7-9], as well as the transfer of structural changes and information along the DNA molecule [10]. The role of the

\footnotetext{
*yakushev@icb.psn.ru

*k*k1983@mail.ru
} 
dynamic properties of the open states in the prediction of bacterial promoters has been recently considered by [11].

The DNA open states are often modeled by kinks which are one-soliton solutions of the sine-Gordon equation [12-15]. Recent advances in the studies of the kinks dynamics are associated with the McLaughlin-Scott equation [16], well known in nonlinear physics and successfully applied for homogeneous synthetic DNA [17], and the block method [18] that allows extending the McLaughlin-Scott approach to the case of heterogeneous sequences [1921].

In this paper, we apply both methods for modeling dynamics of kinks in the plasmid pBR322 that is a small circular DNA widely used in the gene research, and its components are applied to create new instrumental plasmids [22]. The nucleotide sequence of the plasmid, the arrangement of the main functionally important regions in the sequence and its functional properties are well known [23]. However, nothing is known about the internal dynamics of this plasmid, although it could be assumed that the small size and the presence of two coding regions in the matrix chain make the plasmid pBR322 a convenient object for studying the DNA internal mobility.

In the next section we describe shortly the pBR322 sequence and the methods to study the internal dynamics of the plasmid. Then we present the results of calculations of the energy profile of the sequence as well as the time dependences of the kink energy, velocity and coordinate for three different values of the initial velocity: $150 \mathrm{~m} / \mathrm{s}, 1650 \mathrm{~m} / \mathrm{s}$ and $1879 \mathrm{~m} / \mathrm{s}$. In the final section we discuss the approach applied, the obtained results and the conditions for kink passing the entire plasmid.

\section{MATERIALS AND METHODS}

As we mentioned above the plasmid pBR322 is a convenient object to study the DNA nonlinear dynamics. Schematic representation of the plasmid is shown in Fig. 1,a. The linear version of the scheme that is obtained by cutting the circular DNA at the point $\mathrm{S}$ (located at the middle of the unique EcoRI site) is shown in Fig. 1,b. The nucleotide sequence of the plasmid pBR322 (Genbank accession number J01749.1) is completely deciphered [23]. In particular, it is known that the main chain of the plasmid contains two coding regions CDS-1 and CDS-2 with coordinates (86...1276) and (1915...2106), respectively, and the complementary chain contains one coding region CDS-3 with coordinates $(3293 \ldots 4153)$.

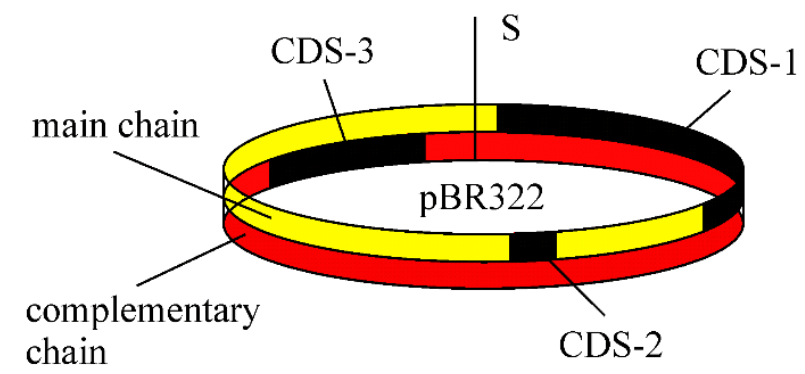

a

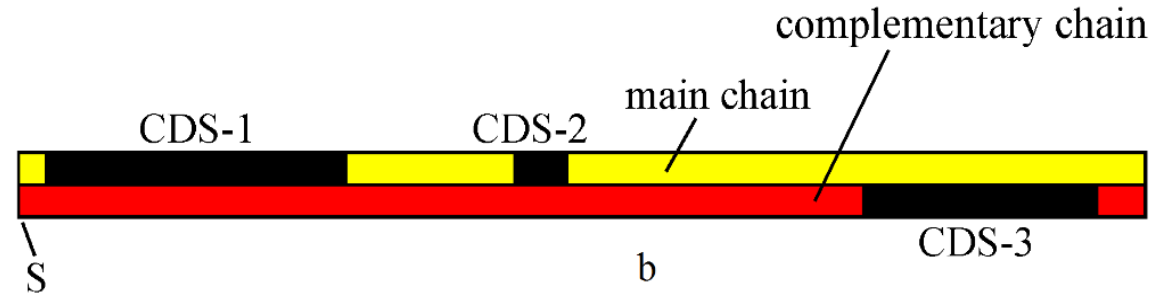

Fig. 1. Schematic picture of the plasmid pBR32 (a) and its linear analogue (b). S is the cut point. The coding regions CDS-1, CDS-2 and CDS-3 are indicated in black. The CDS-1 and CDS-2 regions are located in the main polynucleotide chain, and the CDS-3 region is located in the complementary chain. 


\section{Theoretical model and methods used}

To model the kink movement in the plasmid, we use the McLaughlin-Scott equation [16] adapted for homogeneous DNA in [17]:

$$
\frac{d \mathrm{v}_{k}(t)}{d t}=-\frac{\beta}{I} \mathrm{v}_{k}(t)\left[1-\left(\frac{\mathrm{v}_{k}(t)}{C}\right)^{2}\right]
$$

This equation determines the velocity of the kink $v_{k}(t)$. Here $\beta=\alpha R^{2}, \alpha$ is the coefficient of dissipation, $R$ is the distance between the center of mass of the base and the nearest sugarphosphate chain, $I$ is the moment of inertia of the base, $C=\left(K^{\prime} a^{2} / I\right)^{1 / 2}$ is the sound velocity in DNA, $K^{\prime}$ is the torsion rigidity of the sugar-phosphate chain, $a$ is the distance between the nearest base pairs.

The solution of Eq. (1) has the form [17]:

$$
\mathrm{v}_{k}(t)=\frac{\mathrm{v}_{0} \gamma_{0} \exp \left(-\frac{\beta}{I}\left(t-t_{0}\right)\right)}{\sqrt{1+\left(\frac{\mathrm{v}_{0} \gamma_{0}}{C}\right)^{2} \exp \left(-\frac{2 \beta}{I}\left(t-t_{0}\right)\right)}},
$$

where $v_{0}$ is the value of the kink velocity at the initial time $t_{0}, \gamma_{0}=\left(1-v_{0} / C^{2}\right)^{-1 / 2}$.

In our calculations, we use also the solution for the kink coordinate [19]:

$$
z_{k}(t)=z_{0}-C \frac{I}{\beta} \operatorname{arcsinh}\left(\frac{\mathrm{v}_{0}}{C} \gamma_{0} \exp \left(-\frac{\beta}{I}\left(t-t_{0}\right)\right)\right)+C \frac{I}{\beta} \operatorname{arcsinh}\left(\frac{\mathrm{v}_{0}}{C} \gamma_{0}\right)
$$

and the formula for the total kink energy:

$$
E_{k}(t)=\frac{E_{0}}{\sqrt{1-\frac{v_{k}^{2}(t)}{C^{2}}}}
$$

Here $E_{0}=8 \sqrt{K^{\prime} V}$ is the rest energy of the kink, $V$ is the parameter that characterizes interaction between bases in pairs, $z_{0}$ is the kink coordinate at the initial time $t_{0}$.

To extend the applicability of Eq. (1) and formulas (2)-(4) to inhomogeneous sequences, we use the block method. In accordance with the method, we divide the main sequence of the plasmid pBR322 into several regions (blocks). As can be seen from Fig. 1b, the number of the blocks in the case of the pBR322 sequence is equal to five: two coding regions (CDS-1 and CDS-2) and three intermediate regions with coordinates: (1..85), (1277..1914) and (2107..4361). Then we assign the numbers to the blocks, as shown in Fig. 2.

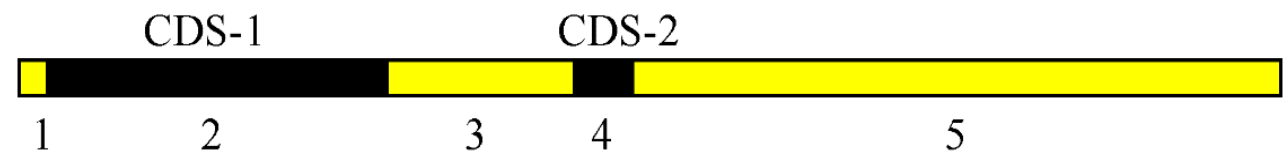

Fig. 2. The blocks numbering in the main chain of the plasmid pBR322.

The data on the structure of each of these blocks are presented in Table 1 where $N^{(i)}$ is the total number of bases in the $i$-th block, $N_{A}^{(i)}$ is the number of adenines in the $i$-th block, 
$N_{T}^{(i)}$ is the number of thymines in the $i$-th block, $N_{G}^{(i)}$ is the number of guanines in the $i$-th block, $N_{C}^{(i)}$ is the number of cytidines in the $i$-th block, $i=1,2,3,4,5$.

Table 1. The details of the block structure of the pBR322 sequence

\begin{tabular}{|c|c|c|c|c|c|c|}
\hline $\begin{array}{c}\text { Block } \\
\text { number }\end{array}$ & $\begin{array}{c}\text { Block } \\
\text { coordinates }\end{array}$ & $N_{A}^{(i)}$ & $N_{T}^{(i)}$ & $N_{G}^{(i)}$ & $N_{C}^{(i)}$ & $N^{(i)}$ \\
\hline 1 & $(1 . .85)$ & 597 & 584 & 573 & 586 & 2340 \\
\hline $2($ CDS-1) & $(86.1276)$ & 190 & 268 & 353 & 380 & 1191 \\
\hline 3 & $(1277 . .1914)$ & 142 & 145 & 160 & 191 & 638 \\
\hline $4($ CDS-2) & $(1915 . .2106)$ & 54 & 37 & 48 & 53 & 192 \\
\hline 5 & $(2107 . .4361)$ & 597 & 584 & 573 & 586 & 2340 \\
\hline
\end{tabular}

In the case of homogeneous sequence, the coefficients of Eq. (1) are constants. In the case of heterogeneous sequence, the coefficients depend on the nucleotide sequence. To take into account this dependence with the help of the block method, we average the main DNA parameters within each of the blocks:

$$
\begin{aligned}
& \tilde{I}^{(i)}=I_{A} C_{A}^{(i)}+I_{T} C_{T}^{(i)}+I_{G} C_{G}^{(i)}+I_{C} C_{C}^{(i)}, \\
& \tilde{K}^{\prime(i)}=K_{A}^{\prime} C_{A}^{(i)}+K_{T}^{\prime} C_{T}^{(i)}+K_{G}^{\prime} C_{G}^{(i)}+K_{C}^{\prime} C_{C}^{(i)}, \\
& \tilde{V}^{(i)}=V_{A} C_{A}^{(i)}+V_{T} C_{T}^{(i)}+V_{G} C_{G}^{(i)}+V_{C} C_{C}^{(i)}, \\
& \tilde{\beta}^{(i)}=\beta_{A} C_{A}^{(i)}+\beta_{T} C_{T}^{(i)}+\beta_{G} C_{G}^{(i)}+\beta_{C} C_{C}^{(i)},
\end{aligned}
$$

where $C_{j}^{(i)}$ is the concentration of nitrous bases of the $j$-th type in the $i$-th block $(j=\mathrm{A}, \mathrm{T}, \mathrm{G}, \mathrm{C})$. The values of the parameters presented in the right-hand parts of formulas (5) are shown in Table 2.

Table 2. Parameters of DNA [13]

\begin{tabular}{|c|c|c|c|c|}
\hline $\begin{array}{c}\text { Type of the } \\
\text { base }\end{array}$ & $\begin{array}{c}I_{i} \times 10^{-44} \\
\left(\mathrm{~kg} \cdot \mathrm{m}^{2}\right)\end{array}$ & $\begin{array}{c}V_{i} \times 10^{-20} \\
(\mathrm{~J})\end{array}$ & $\begin{array}{c}K_{i}^{\prime} \times 10^{-18} \\
(\mathrm{~J})\end{array}$ & $\begin{array}{c}\beta_{i} \times 10^{-34} \\
(\mathrm{~J} \cdot \mathrm{s})\end{array}$ \\
\hline $\mathrm{A}$ & 7.61 & 2.09 & 2.35 & 4.25 \\
\hline $\mathrm{T}$ & 4.86 & 1.43 & 1.61 & 2.91 \\
\hline $\mathrm{G}$ & 8.22 & 3.12 & 2.27 & 4.10 \\
\hline $\mathrm{C}$ & 4.11 & 2.12 & 1.54 & 2.79 \\
\hline
\end{tabular}

The results of the averaging are presented in Table 3.

Table 3. The averaged coefficients of Eq. (1)

\begin{tabular}{|c|c|c|c|c|c|}
\hline $\begin{array}{c}\text { Block } \\
\text { number }\end{array}$ & $\begin{array}{c}\text { Block } \\
\text { coordinates }\end{array}$ & $\begin{array}{c}\tilde{I}^{(i)} \times 10^{-44} \\
\left(\mathrm{~kg} \cdot \mathrm{m}^{2}\right)\end{array}$ & $\begin{array}{c}\tilde{V}^{(i)} \times 10^{-2} \\
(\mathrm{~N} / \mathrm{m})\end{array}$ & $\begin{array}{c}\tilde{K}^{\prime(i)} \times 10^{-18} \\
(\mathrm{~N} \cdot \mathrm{m})\end{array}$ & $\begin{array}{c}\tilde{\beta}^{(i)} \times 10^{-34} \\
(\mathrm{~J} \cdot \mathrm{c})\end{array}$ \\
\hline 1 & $(1 . .85)$ & 6.19 & 2.18 & 1.94 & 3.51 \\
\hline $2(\mathrm{CDS}-1)$ & $(86 . .1276)$ & 6.05 & 2.26 & 1.90 & 3.44 \\
\hline 3 & $(1277 . .1914)$ & 6.09 & 2.21 & 1.92 & 3.47 \\
\hline $4(\mathrm{CDS}-2)$ & $(1915 . .2106)$ & 6.26 & 2.23 & 1.96 & 3.55 \\
\hline 5 & $(2107 . .4361)$ & 6.19 & 2.18 & 1.94 & 3.51 \\
\hline
\end{tabular}

Taking into account the relations (5) we can write formulas determining the kink velocity, coordinate and total energy in each of the five blocks, in the following form: 


$$
\begin{gathered}
v_{k}^{(i)}(t)=\frac{v_{0}^{(i)} \tilde{\gamma}_{0}^{(i)} \exp \left(-\frac{\tilde{\beta}^{(i)}}{\tilde{I}^{(i)}}\left(t-t_{0 i}\right)\right)}{\sqrt{1+\left(\frac{v_{0}^{(i)} \tilde{\gamma}_{0}^{(i)}}{\tilde{C}^{(i)}}\right)^{2} \exp \left(-\frac{2 \tilde{\beta}^{(i)}}{\tilde{I}^{(i)}}\left(t-t_{0 i}\right)\right)}} \\
z_{k}^{(i)}(t)=z_{0}^{(i)}-\tilde{C}^{(i)} \frac{\tilde{I}^{(i)}}{\tilde{\beta}^{(i)}} \operatorname{arcsinh}\left(\frac{v_{0}^{(i)}}{\tilde{C}^{(i)}} \tilde{\gamma}_{0}^{(i)} \exp \left(-\frac{\tilde{\beta}^{(i)}}{\tilde{I}^{(i)}}\left(t-t_{0 i}\right)\right)\right)+\tilde{C}^{(i)} \frac{\tilde{I}^{(i)}}{\tilde{\beta}^{(i)}} \operatorname{arcsinh}\left(\frac{v_{0}^{(i)}}{\tilde{C}^{(i)}} \tilde{\gamma}_{0}^{(i)}\right), \\
\tilde{E}_{k}^{(i)}(t)=\frac{8 \sqrt{\tilde{K}^{(i)} \tilde{V}^{(i)}}}{\sqrt{1-\left(\frac{v_{k}^{(i)}(t)}{\tilde{C}^{(i)}}\right)^{2}}},
\end{gathered}
$$

where $v_{0}^{(i)}$ is the initial velocity of the kink in the $i$-th block, $\tilde{C}^{(i)}=\left(\tilde{K}^{\prime(i)} a^{2} / \tilde{I}^{(i)}\right)^{1 / 2}$ is the sound velocity in the $i$-th block, $a=3.4 \times 10^{-10} \mathrm{~m}$ is the distance between base pairs, $t_{0 i}$ is the start time of the motion of the kink on the $i$-the block, $z_{0}^{(i)}$ is the kink coordinate at $t_{0 i}, i=1$, $2,3,4,5$.

To obtain the time dependences of the kink velocity, coordinate and total energy within the whole sequence of the plasmid, it is necessary to "stitch" the solutions (6)-(8) at the boundaries between the blocks. If we suggest that at the crossing the boundaries the loss of the kink energy is absent, the "stitching" is reduced to a simple equalization of the total kink energy to the left and to the right of the boundary.

\section{RESULTS AND DISCUSSION}

\section{Energy profile of the plasmid pBR322}

If the initial kink velocity is less than the velocity of sound in DNA, the total energy (8) can be expanded in a series in the small parameter $\left(v_{k}^{(i)}(t) / \tilde{C}^{(i)}\right)$

$$
\tilde{E}^{(i)}(t)=\tilde{E}_{0}^{(i)}\left[1+\frac{\left(\mathrm{v}_{k}^{(i)}(t)\right)^{2}}{2\left(\tilde{C}^{(i)}\right)^{2}}\right]
$$

So, in this context the rest energy $\tilde{E}_{0}^{(i)}$ can be interpreted as a potential energy of the kink in the $i$-th block.

To construct the energy profile of the plasmid pBR322, we calculated the rest energy of the kink $\tilde{E}_{0}^{(i)}=8 \sqrt{\tilde{K}^{\prime(i)} \tilde{V}^{(i)}}$ for each of the blocks. When making the calculations, it was taken into account that the plasmid has the form of a ring, and therefore for the 1-st and 5-th blocks the coefficients averaged over the combined block $5+1$ were used. The results of the rest energies calculations are presented in Table 4.

Table 4. Kink rest energy, calculated for each of the five blocks

\begin{tabular}{|c|c|}
\hline Block number & $\tilde{E}_{0}^{(i)} \times 10^{-20}(\mathrm{~J})$ \\
\hline 1 & 164.82 \\
\hline 2 (CDS- 1$)$ & 165.71 \\
\hline 3 & 164.66 \\
\hline $4($ CDS-2) & 167.35 \\
\hline 5 & 164.82 \\
\hline
\end{tabular}


With the help of the data from Table 4 we constructed the energy profile of the main sequence of the plasmid (Fig. 3). The horizontal scale shows the coordinates of the nitrogen bases in the units of base pairs (bp), $1 \mathrm{bp}=3.4 \times 10^{-10} \mathrm{~m}$. Having the energy profile of the plasmid, we got the opportunity to transform the problem of the kink movement in the plasmid to the problem of the movement of a quasi particle in the potential field with the obtained energy profile.

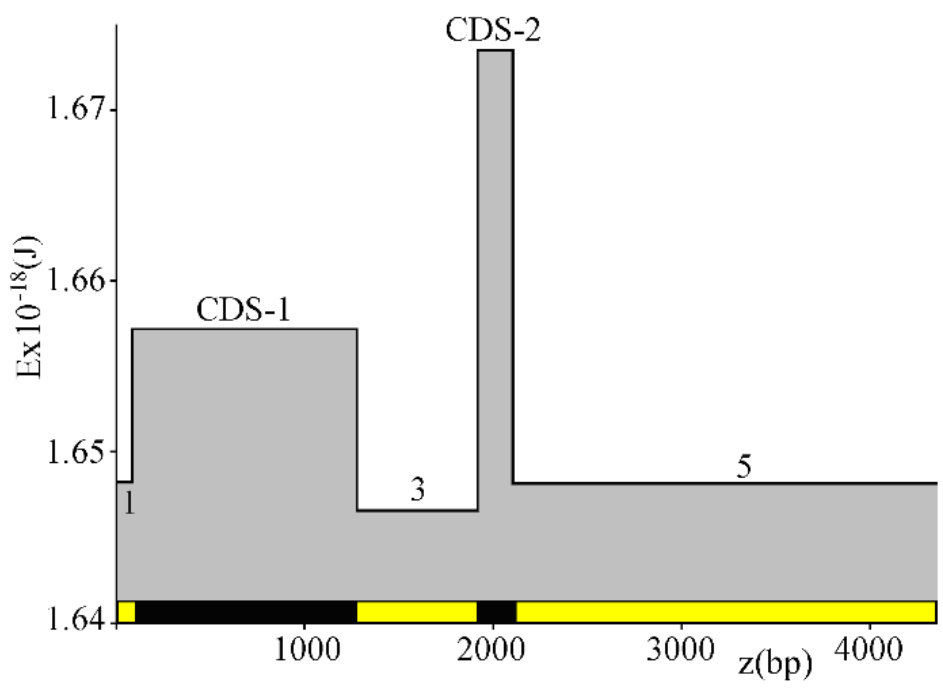

Fig. 3. Energy profile of the plasmid pBR322.

As it can be seen from Fig. 3, the energy profile contains two barriers that correspond to the coding regions CDS-1 and CDS-2. This allows us to suggest that the behavior of the kink in the plasmid pBR322 in many respects is similar to the behavior of a quasi particle and is determined by whether the kink can overcome the barriers or not, and the latter will depend on the initial velocity of the kink.

\section{Kink velocity in the plasmid pBR322}

To construct the graphs of the time dependence of the kink velocity, we have used formula (6), the parameters from Table 3 and the conditions of "stitching" described above. Calculations were carried out for three values of the initial kink velocity: $150 \mathrm{~m} / \mathrm{s}, 1650 \mathrm{~m} / \mathrm{s}$ and $1879 \mathrm{~m} / \mathrm{s}$, although it should be noted that the algorithm described above is valid for any values of the initial kink velocities which are less than the sound velocity in the first block $\left(\tilde{C}^{(1)}=1904.60 \mathrm{~m} / \mathrm{s}\right)$.

These three model values were selected as follows. Preliminary we made estimations of the two minimum values of the initial kink velocities necessary to overcome the first and second energy barriers. The minimum initial kink velocity necessary to overcome the first barrier was calculated using the formula:

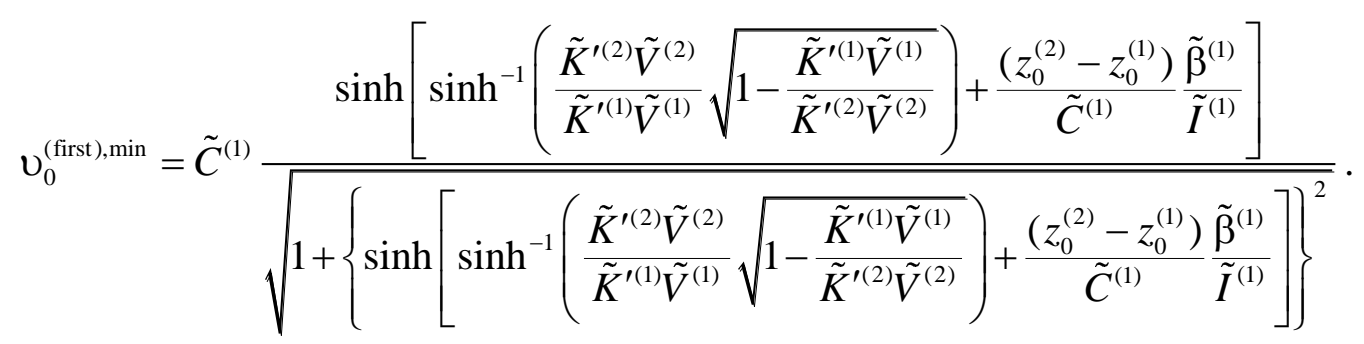


The result obtained was equal to $v_{0}^{\text {(first),min }}=359.20 \mathrm{~m} / \mathrm{s}$. We name it the first minimum value of the initial velocity.

Formula (9) has been obtained as follows. At the beginning, we found the minimum kink velocity to the left of the 1-st boundary $v_{\text {end }}^{\text {(first),min }}$. It was done by equating the total kink energy to the left of the boundary and the minimum kink energy (i.e. the kink rest energy) to the right of this boundary:

$$
v_{\text {end }}^{(\text {first }) \text { min }}=\tilde{C}^{(1)} \sqrt{1-\frac{\tilde{K}^{(1)} \tilde{V}^{(1)}}{\tilde{K}^{(2)} \tilde{V}^{(2)}}} .
$$

On the other hand, the velocity $v_{\text {end }}^{\text {(first),min }}$ can be obtained in another way. To do this, we took formula (6) and rewrote it for the case considered:

$$
v_{\text {end }}^{\text {(first),min }}=\frac{v_{0}^{(1), \min } \tilde{\gamma}_{0}^{(1), \min } \exp \left(-\frac{\tilde{\beta}^{(1)}}{\tilde{I}^{(1)}}\left(t_{1}^{\text {min }}-t_{01}\right)\right)}{\sqrt{1+\left[\left(\frac{v_{0}^{(1), \min }}{\tilde{C}^{(1)}} \tilde{\gamma}_{0}^{(1), \min }\right) \exp \left(-\frac{\tilde{\beta}^{(1)}}{\tilde{I}^{(1)}}\left(t_{1}^{\min }-t_{01}\right)\right)\right]^{2}}} .
$$

Here $t_{1}^{\min }$ is the right boundary of the first time interval. The value of $t_{1}^{\min }$ is determined by the formula:

$$
t_{1}^{\min }=t_{01}-\frac{\tilde{I}^{(1)}}{\tilde{\beta}^{(1)}} \ln \left\{\frac{\tilde{C}^{(1)}}{v_{0}^{(1), \min } \tilde{\gamma}_{0}^{(1), \min }} \sinh \left[\sinh ^{-1}\left(\frac{v_{0}^{(1), \min }}{\tilde{C}^{(1)}} \tilde{\gamma}_{0}^{(1), \text { min }}\right)-\frac{\left(z_{0}^{(2)}-z_{0}^{(1)}\right)}{\tilde{C}^{(1)}} \frac{\tilde{\beta}^{(1)}}{\tilde{I}^{(1)}}\right]\right\}
$$

which is easily derived from (7). Inserting this formula for $t_{1}^{\min }$ into (11) we find formula for $\mathrm{v}_{\text {end }}^{(\text {first }) \text { min }}$ :

$$
v_{0}^{(\text {first }) \text { min }}=\frac{\tilde{C}^{(1)} \sinh \left[\sinh ^{-1}\left(\frac{v_{0}^{(1), \min }}{\tilde{C}^{(1)}} \tilde{\gamma}_{0}^{(1), \min }\right)+\frac{\left(z_{0}^{(2)}-z_{0}^{(1)}\right)}{\tilde{C}^{(1)}} \frac{\tilde{\beta}^{(1)}}{\tilde{I}^{(1)}}\right]}{\sqrt{1+\left\{\sinh \left[\sinh ^{-1}\left(\frac{v_{0}^{(1), \min }}{\tilde{C}^{(1)}} \tilde{\gamma}_{0}^{(1), \min }\right)+\frac{\left(z_{0}^{(2)}-z_{0}^{(1)}\right)}{\tilde{C}^{(1)}} \frac{\tilde{\beta}^{(1)}}{\tilde{I}^{(1)}}\right]\right\}^{2}}} .
$$

Then, by equating formulas (10) and (12) we obtained the desired formula (9).

Using a similar formula

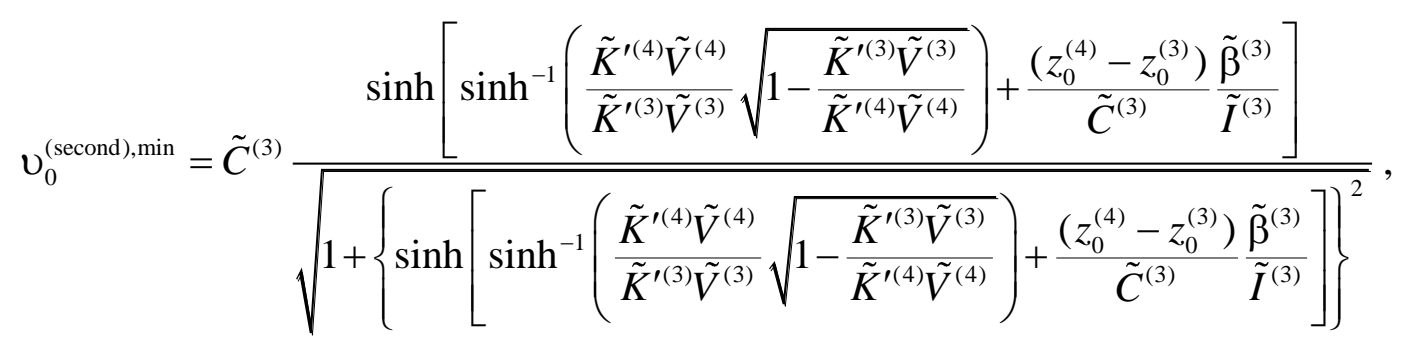

we obtained the second minimum value of the initial kink velocity ( $v_{0}^{(\text {second), } \min }=1656.66 \mathrm{~m} / \mathrm{s}$ ) that is necessary to overcome the other energy barrier.

Considering these two estimations, we selected up the first model value of the initial velocity $(150 \mathrm{~m} / \mathrm{s})$, so that it was less than the first minimum velocity $\left(U_{0}^{(\text {first }), \min }=359.20 \mathrm{~m} / \mathrm{s}\right)$. In this case, it can be expected that the kink will stop within the 1-st block, not reaching the coding region CDS- 1 . 
The second model value of the initial velocity $(1650 \mathrm{~m} / \mathrm{s})$, was chosen so that it was greater than the first minimum velocity $\left(v_{0}^{(\text {first }) \text {,min }}=359.20 \mathrm{~m} / \mathrm{s}\right)$, but less than the second minimum velocity $\left(v_{0}^{\text {(second),min }}=1656.66 \mathrm{~m} / \mathrm{s}\right)$. In this case, kink will overcome the first energy barrier (CDS-1), but will not reach the second barrier (CDS-2).

The third model value of the initial velocity $(1879 \mathrm{~m} / \mathrm{s})$ was chosen so that it was greater than the second minimum velocity $\left(v_{0}^{\text {(second),min }}=1656.66 \mathrm{~m} / \mathrm{s}\right.$ ), but less than the sound velocity ( $\tilde{C}^{(1)}=1904.60 \mathrm{~m} / \mathrm{s}$ ). It can be expected that in this case the kink will overcome both the first (CDS-1) and the second (CDS-2) energy barriers.

The results of calculating the time dependence of the kink velocity are shown in Fig. 4.

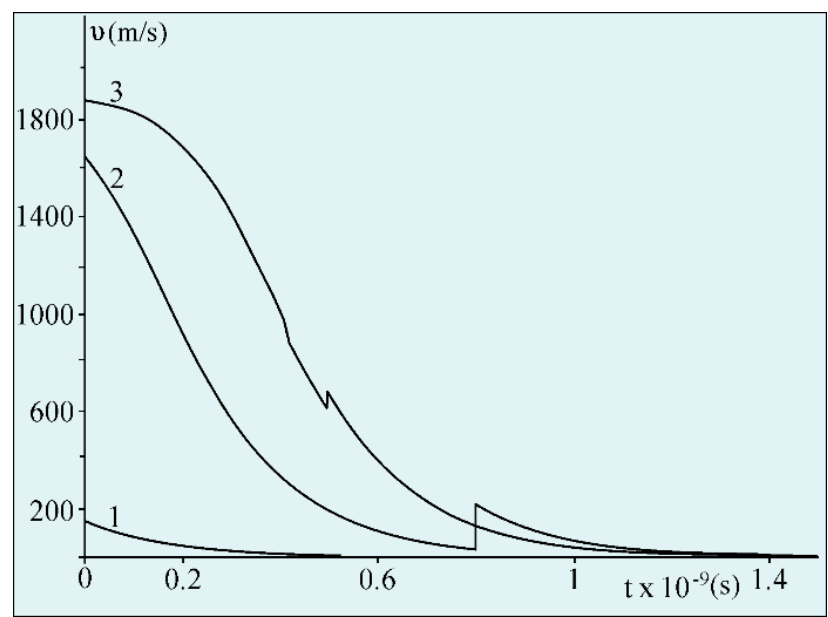

Fig. 4. Time dependence of the kink velocity in the plasmid pBR322. The curves 1,2 and 3 correspond to initial velocities of $150 \mathrm{~m} / \mathrm{s}, 1650 \mathrm{~m} / \mathrm{s}$ and $1879 \mathrm{~m} / \mathrm{s}$, respectively.

The three curves in Fig. 4 show the decrease of the kink velocity that is explained by effects of dissipation. The smooth curve 1 corresponds to the case when the initial kink velocity is small and kink stops inside the 1-st block. The curve 2 has two zigzags: one unnoticeable zigzag with the negligibly small change of the kink velocity equal to $\sim 2 \mathrm{~m} / \mathrm{c}$ at $t_{1}^{\text {end, } 1650}=1.77 \times 10^{-11} \mathrm{~s}$ (this time corresponds to the moment of crossing the boundary between the 2-nd and 3-rd blocks) and one noticeable zigzag with the change of the kink velocity equal to $\sim 183 \mathrm{~m} / \mathrm{c}$ at $t_{2}^{\text {end, } 1650}=7.99 \times 10^{-10} \mathrm{~s}$ (this time corresponds to the moment of crossing the boundary between the 2-nd and 3-rd blocks). The curve 3 has four zigzags: one unnoticeable zigzag with the negligibly small change of the kink velocity changes equal to $\sim 1 \mathrm{~m} / \mathrm{c}$ at $t_{1}^{\text {end,1879 }}=1.54 \times 10^{-11} \mathrm{~s}$ (this time corresponds to the moment of crossing the boundary between the 1-nd and 2-nd blocks), one unnoticeable zigzag with the negligibly small change of the kink velocity changes equal to $\sim 7 \mathrm{~m} / \mathrm{c}$ at $t_{2}^{\text {end,1879 }}=2.43 \times 10^{-10} \mathrm{~s}$ (this time corresponds to the moment of crossing the boundary between the 2-nd and 3-nd blocks), one noticeable zigzag with the change of the kink velocity changes equal to $\sim 50 \mathrm{~m} / \mathrm{c}$ at $t_{3}^{\text {end,1879 }}=4.10 \times 10^{-10} \mathrm{~s}$ (this time corresponds to the moment of crossing the boundary between the 3-nd and 4-nd blocks) and one noticeable zigzag with the change of the kink velocity changes equal to $\sim 76 \mathrm{~m} / \mathrm{c}$ at $t_{4}^{\text {end,1879 }}=4.96 \times 10^{-10} \mathrm{~s}$ (this time corresponds to the moment of crossing the boundary between the 4-nd and 5-nd blocks). Thus, our assumption that the kink behavior is determined by whether the kink is able to overcome the energy barriers or not, and that the overcoming of the barriers depends on the initial kink velocity, is fully confirmed. 


\section{Kink coordinates in the plasmid pBR322}

Formula (7) and parameter values from Table 3 have been used to calculate the time dependence of the kink coordinate for three values of the initial velocity: $150 \mathrm{~m} / \mathrm{s}, 1650 \mathrm{~m} / \mathrm{s}$ and $1879 \mathrm{~m} / \mathrm{s}$. The results of the calculation are presented in Fig. 5.

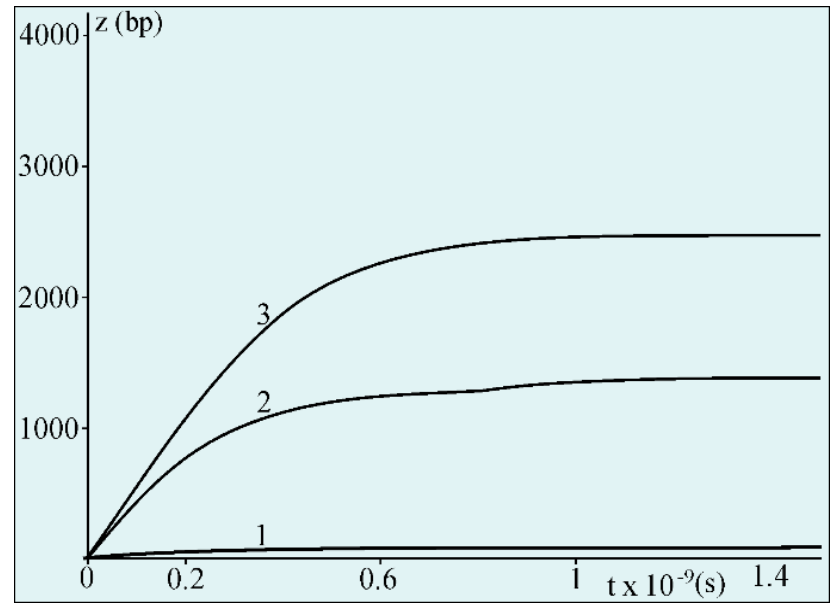

Fig. 5. Time dependence of the kink coordinate in the plasmid pBR322. The curves 1,2 and 3 correspond to initial velocities of $150 \mathrm{~m} / \mathrm{s}, 1650 \mathrm{~m} / \mathrm{s}$ and $1879 \mathrm{~m} / \mathrm{s}$, respectively.

In Fig. 5 all three curves smoothly grow and reach the stationary values corresponding to the points of the kink stop. We calculated the coordinates of these points for different values of the initial kink velocity. The results are: $z_{0}^{(1) \text {,stop, } 150}=77 \mathrm{bp}, z_{0}^{(1) \text {,stop, } 1650}=1368 \mathrm{bp}$ and $z_{0}^{(1), s t o p, 1879}=2477$ bp. From Fig. 5 it can be seen that the greater the initial kink velocity, the more path of the kink till the complete stop.

\section{Kink total energy in the plasmid pBR322}

To construct the time dependence of the total energy of the kink for different values of the initial velocity, we used formula (8) and parameter values from Table 3.

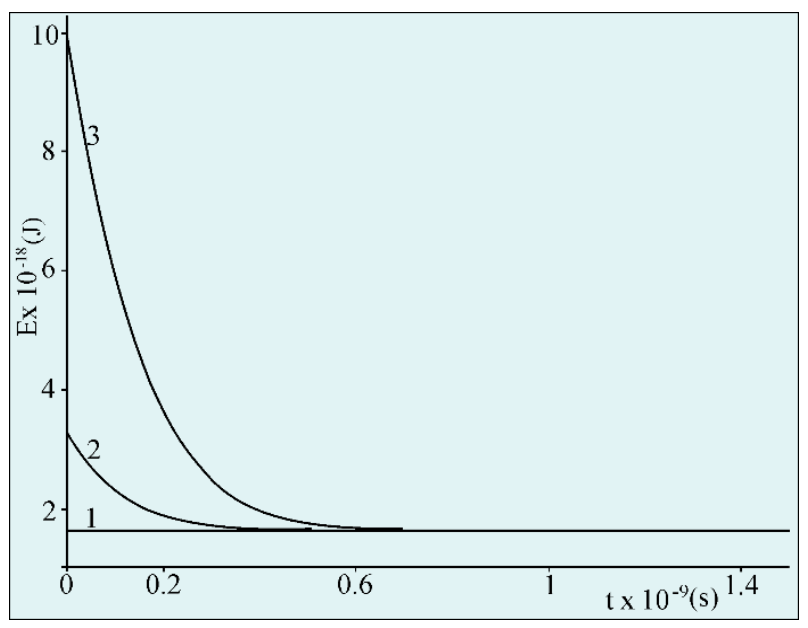

Fig. 6. Time dependence of the total energy of the kink in the plasmid pBR322. The curves 1, 2 and 3 correspond to initial velocities of $150 \mathrm{~m} / \mathrm{s}, 1650 \mathrm{~m} / \mathrm{s}$ and $1879 \mathrm{~m} / \mathrm{s}$, respectively.

The three curves shown in Fig. 6 gradually decrease with time and reach the values equal to the kink rest energies $164.82 \times 10^{-20}(\mathrm{~J}), 164.66 \times 10^{-20}(\mathrm{~J})$ and $164.82 \times 10^{-20}(\mathrm{~J})$. 


\section{Kink trajectories on the phase plane}

To calculate kink trajectories on the phase plane $\{v, z\}$, it is convenient to rewrite equation (1) in the form of the two coupled ordinary differential equations for the velocity and coordinate of the kink:

$$
\begin{gathered}
\frac{d z_{k}^{(i)}(t)}{d t}=v_{k}^{(i)}(t) \\
\frac{d v_{k}^{(i)}(t)}{d t}=\frac{\tilde{\beta}^{(i)}}{\tilde{I}^{(i)}} v_{k}^{(i)}(t)\left[1-\left(\frac{v_{k}^{(i)}(t)}{\tilde{C}^{(i)}}\right)^{2}\right]
\end{gathered}
$$

where index $i$ denotes the number of the block $(i=1,2, \ldots 5)$. Results of numerical calculations of the kink trajectories in each of the blocks and of "stitching" the obtained solutions at the boundaries between the blocks are shown in Fig. 7.

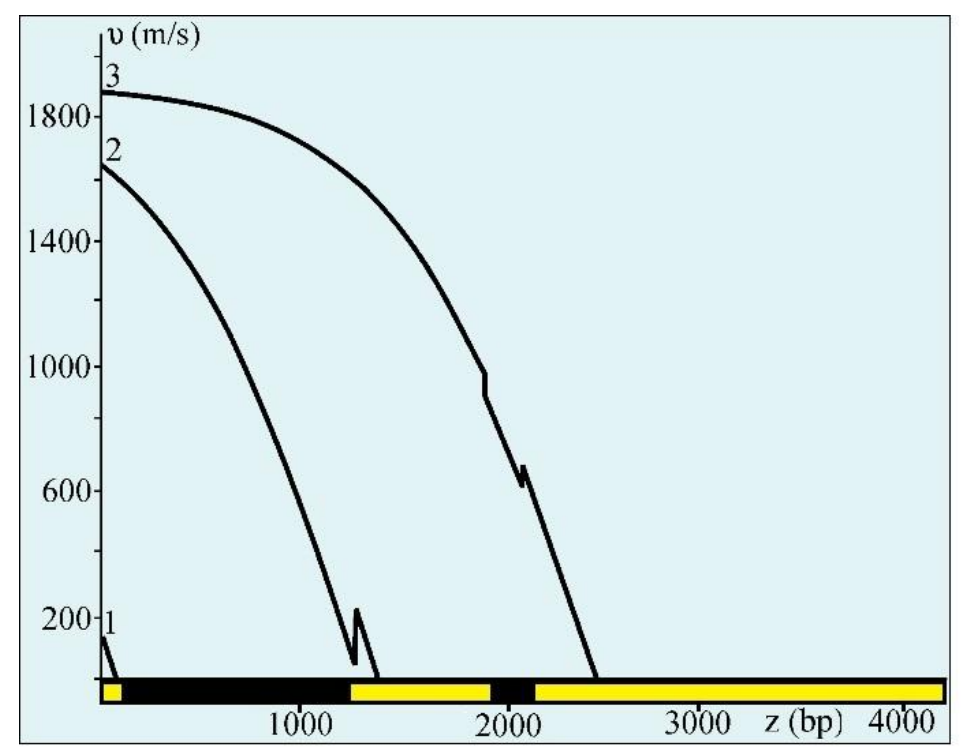

Fig. 7. Kink trajectories on the phase plane $\{v, z\}$. The curves 1,2 and 3 correspond to initial velocities of $150 \mathrm{~m} / \mathrm{s}, 1650 \mathrm{~m} / \mathrm{s}$ and $1879 \mathrm{~m} / \mathrm{s}$, respectively.

It is interesting to note that the phase trajectories shown in Fig.7, demonstrate the same behavior as those obtained in Fig. 4. Indeed, the curve 1 is smooth, the curve 2 has one noticeable zigzag at $z=1277 \mathrm{bp}$ and the curve 3 has two noticeable zigzags at $z=1915 \mathrm{bp}$ and at $z=2107 \mathrm{bp}$.

\section{CONCLUSIONS}

In the present work, we investigated the dynamic behavior of local conformational distortions - kinks, in the plasmid pBR322 by the methods of mathematical modeling. In comparison with other studies, our approach has two advantages. First, we considered the internal dynamics of the pBR322 sequence that has not been considered before. Second, instead of the quasi-homogeneous approximation we applied the block method that gave a possibility to take into account the arrangement of the CDS regions in the sequence.

We calculated the energy profile of the pBR322 sequence having two energy barriers (CDS-1 and CDS-2), and interpreted the movement of the kinks in the plasmid as the movement of a quasi particles in the potential field with this profile. In the earlier approaches based on the quasi-homogeneous approximation, there was not a possibility to obtain any profile. 
To model the structure of the pBR322 sequence, we used five blocks. However, the number of the blocks can be increased to take into account more details of the sequence structure. When doing this, it is necessary to keep in mind one important condition. The block size should exceed the size of the kink, i.e. the block length should be more than 10-15 bp.

We calculated the main characteristics of the kink movement: the time dependences of the kink velocity, coordinate and total energy for three values of the initial velocity: $150 \mathrm{~m} / \mathrm{s}$, $1650 \mathrm{~m} / \mathrm{s}$ and $1879 \mathrm{~m} / \mathrm{s}$, which were chosen to avoid the reflections from the energy barriers (CDS-1 and CDS-2). Our assumption that kink behavior is determined by whether the kink is able to overcome the energy barriers or not, and that the overcoming of the barriers depends on the initial kink velocity has been fully confirmed. We found also that the necessary condition for kink passing the entire plasmid was the enough large value of the initial kink velocity: $v \geq 1656.66 \mathrm{~m} / \mathrm{c}$.

Despite the fact that we have considered here only three values of the initial kink velocity, the approach proposed can be applied to any values of the initial kink velocity. Only one requirement should be fulfilled: the initial kink velocity should be less than the DNA sound velocity. This condition arises from mathematical requirements of the existence and stability of the solution in the form of kink.

We would like to note that the obtained results on the kink velocity, coordinates and phase trajectories can be presented in a more compact and convenient form of $3 \mathrm{D}$ trajectories as it is shown in Fig. 8.

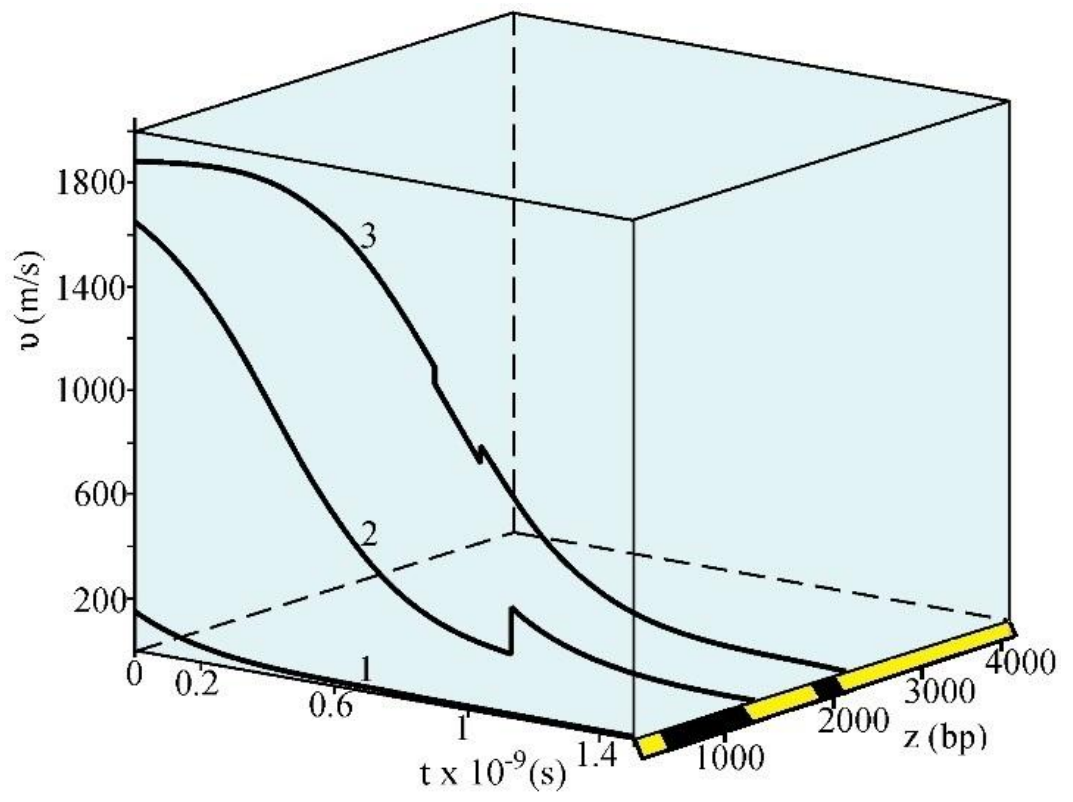

Fig. 8. 3D trajectories of the kinks in the plasmid pBR322. The curves 1,2 and 3 correspond to initial velocities of $150 \mathrm{~m} / \mathrm{s}, 1650 \mathrm{~m} / \mathrm{s}$ and $1879 \mathrm{~m} / \mathrm{s}$, respectively.

This presentation permits to interpret the results shown in Fig. (4), (5) and (7) as the projections of the kink 3D trajectories on the planes $\{v, t\},\{z, t\}$ and $\{v, z\}$, respectively.

It should be noted, however, that these results on the kink dynamics were obtained under two additional constraints. First, we used a simplified model that took into account the mobility of the nitrous bases in one of the two polynucleotide chains of DNA, the second chain being modeled only as an average field. Second, to calculate the dynamic characteristics of the kink, we used the McLaughlin-Scott equation which is valid only for the case of small dissipation. In spite of these limitations, we expect that the results obtained by simple and convenient block method, as well as by McLaughlin-Scott equation, correctly convey the basic laws of the kink dynamic behavior in plasmid pBR322 and can be applied to another DNA sequences. 


\section{REFERENCES}

1. Englander S.W., Kallenbach N.R., Heeger A.J., Krumhansl J.A., Litwin A. Nature of the open state in DNA structure. Proc. Natl. Acad. Sci. 1980. V. 77. P. 7222-7226.

2. Clark D., Pazdernik N. Biotechnology. 2nd Edition. Academic Cell, 2015.

3. Zuo Y., Steitz T.A. A structure-based kinetic model of transcription. Transcription. 2017. V. 8. P. 1-8. doi: 10.1080/21541264.2016.1234821.

4. He Y., Yan C., Fang J., Inouye C., Tjian R., Ivanov I., Nogales E. Near-atomic resolution visualization of human transcription promoter opening. Nature. 2016. V. 533. P. 359-365. doi: 10.1038/nature17970.

5. Bailey L.J., Doherty A.J. Mitochondrial DNA replication: a PrimPol perspective. Biochem Soc Trans. 2017. V. 45. P. 513-529. doi: 10.1042/BST20160162.

6. Bleichert F, Botchan M.R., Berger J.M. Mechanisms for initiating cellular DNA replication. Science. 2017. V. 355. P. 215-222. doi: 10.1126/science.aah6317.

7. Sicard F., Destainville N., Manghi M. DNA denaturation bubbles: Free-energy landscape and nucleation/closure rates. J. of Chemical Physics. 2015. V. 142. P. 903910. doi: 10.1063/1.4905668.

8. Shi C., Shang F., Zhou M., Zhang P., Wang Y., Ma C. Triggered isothermal PCR by denaturation bubble-mediated strand exchange amplification. Chem. Commun. 2016. V. 52. P. 11551-11554. doi: 10.1039/C6CC05906F.

9. Singh A.R., Granek R. Manipulation of double-stranded DNA melting by force. Phys. Rev. E. 2017. V. 96. P. 032417-032422. doi: 10.1103/PhysRevE.96.032417.

10. Dwiputra D., Hidayat W., Zen F.P. Nonlinear dynamics of DNA bubble induced by site specific DNA-protein interaction. J. Phys.: Conf. Ser. 2017. V. 856. P. 012005-012009. doi: 10.1088/1742-6596/856/1/011001.

11. Ryasik A., Orlov M., Zykova E., Ermak T., Sorokin A. Bacterial promoter prediction: Selection of dynamic and static physical properties of DNA for reliable sequence classification. J. Bioinf. Comput. Biol. 2018. V. 16. P. 1840003. doi: 10.1142/S0219720018400036.

12. Salerno M. Discrete model for DNA-promoter dynamics. Phys. Rev. A. 1991. V. 44. P. 5292-5297.

13. Yakushevich L.V., Krasnobaeva L.A., Shapovalov A.V., Quintero N.R. One- and TwoSoliton Solutions of the Sine-Gordon Equation as Applied to DNA. Biophysics. 2005. V. 50. P. 450-455.

14. Derks G., Gaeta G. A minimal model of DNA dynamics in interaction with RNApolymerase. Physica D: Nonlinear Phenomena. 2011. V. 240. P. 1805-1817. doi: 10.1017/S0956792511000301.

15. Traverso J.J., Manoranjan V.S., Bishop A.R., Rasmussen K., Voulgarakis N.K. Allostery through protein-induced DNA bubbles. Sci. Rep. 2018. V. 5. P. 9037-9043. doi: $10.1038 /$ srep09037.

16. McLaughlin D.W., Scott A.C. Perturbation analysis of fuxon dynamics. Phys. Rev. A. 1978. V 18. P. 1652-1658.

17. Yakushevich L.V., Krasnobaeva L.A. Effects of dissipation and external fields on the dynamics of conformational distortions in DNA. Biophysics. 2007. V. 52. P. 237-243.

18. Grinevich A.A, Ryasik A.A, Yakushevich L.V. The dynamics of polynucleotide chain consisting of two different homogeneous sequences, divided by interface. Computer Research and Modeling. 2013. V. 5. P. 241-253.

19. Grinevich A.A., Ryasik A.A., Yakushevich L.V. Trajectories of DNA bubles. Chaos, Solitons \& Fractals. 2015. V. 75. P. 62-75. doi: 10.1016/j.chaos.2015.02.009.

20. Yakushevich L.V., Krasnobaeva L.A. Trajectories of the DNA kinks in the sequences containing CDS regions. Mathematical Biology and Bioinformatics. 2017. V. 12. P. 113. doi: $10.17537 / 2017.12 .1$. 
21. Yakushevich L.V., Krasnobaeva L.A. Analytical approaches for exploration of the dynamics of genes having one coding region. Biophysics. 2018. V. 63. P. 41-53. doi: 10.1134/S0006350918010190.

22. Bolivar F, Rodriguez R.L., Greene P.J., Betlach M.C., Heyneker H.L., Boyer H.W., Crosa J.H., Falkow S. Construction and characterization of new cloning vehicles. II. A multipurpose cloning system. Gene 1977. V. 2. P. 95-113.

23. Watson N. A new revision of the sequence of plasmid pBR322. Gene. 1988. V. 70. P. 399-403.

Received 25.03.2019.

Revised 27.05.2019.

Published 20.06.2019. 